\title{
LAMENTACIONES DEL PROFETA JEREMÍAS EN CIUTADELLA DE MENORCA: ESTUDIO Y ANÁLISIS DEL FINAL POLIFÓNICO DE UNA FUENTE PRETRIDENTINA
}

\author{
LAMENTATIONS OF THE PROPHET JEREMIAH IN CIUTADELLA DE \\ MENORCA: STUDY AND ANALYSIS OF THE POLYPHONIC \\ END OF A PRE-TRIDENTINE SOURCE
}

Jaume Escandell Guasch

Institut Menorquí d'Estudis

\section{Resumen}

Este artículo supone una primera aproximación a una fuente musical escrita inédita hasta el momento: tres bifolios sueltos de pergamino conservados en el Archivo Diocesano de Menorca que contienen fragmentos de las Lamentaciones del profeta Jeremías. $\mathrm{Su}$ uso es anterior al Concilio de Trento y presumiblemente se fijaron por escrito durante el siglo XV. Se trata de una versión monódica con la particularidad de que la sección final -correspondiente al capítulo 5 de las Lamentaciones-, reaparece tratada polifónicamente a tres voces siguiendo un lenguaje que se ajusta al procedimiento de fabordón. Se plantea, en primer lugar, una descripción general de las características y el contenido de estos documentos; y en segundo, se incide en el análisis, la organización musical y el tipo de lenguaje de la parte polifónica final.

\section{Palabras clave}

Lamentaciones del profeta Jeremías, Ciutadella de Menorca, análisis, polifonía, fabordón, siglos XV y XVI.

\section{Introducción}

El Archivo Diocesano de Menorca (ADM) custodia tres bifolios de pergamino que originalmente formaban parte de un mismo códice, el cual contenía un juego de Lamentaciones del profeta Jeremías anteriores al Concilio de Trento. Se trata de una versión monódica, pero con una particularidad: la parte final incorpora una serie de versos tratados poli-

\begin{abstract}
This article represents a first approach to a written and so far unpublished musical source: three loose parchment bifolia kept in the Diocesan Archive of Menorca that contain fragments from the Lamentations of Prophet Jeremiah. Their use predate the Council of Trent and presumably were written down during the fifteenth century. This is a monophonic version with the difference that the final section -for Chapter 5 of the Lamentations-, reappears polyphonically in three voices following a language that fits the fauxbourdon procedure. We propose, first of all, an overview of the features and content of these documents, and secondly, we deal with the analysis, the musical organization and the polyphonic language type of the ending part.
\end{abstract}

\section{Keywords}

Lamentations of Prophet Jeremiah, Ciutadella de Menorca, analysis, polyphony, fauxbourdon, XV and XVI centuries.

fónicamente mediante un tipo de lenguaje que claramente se enmarca dentro del procedimiento de fabordón. Pese a que el manuscrito original no se conserva en su integridad, los fragmentos que nos han llegado proporcionan información suficiente para esbozar las características generales de estas Lamentaciones, así como para aproximarnos al funcionamiento de la polivocalidad utilizada en la sección final. 


\section{Descripción general, contenido y organización}

El material que nos ocupa está compuesto por tres bifolios sueltos de pergamino, de características similares y contenido estrechamente relacionado, lo que nos conduce a afirmar que en su momento formaron parte de un único documento de mayores dimensiones. El hecho de que estos tres pergaminos hubiesen servido de tapas de libros durante los siglos XVI y XVII aceleró el deterioro de su contenido original, al mismo tiempo que ocasionó que fuesen recortados para ajustarlos a las medidas de los nuevos fascículos a los cuales se debieron coser. En algunas caras aparecen numerosas anotaciones de contabilidad y, como fecha más antigua, se puede leer al 31 de desembre 1569. Pese a que inicialmente formaban parte de un único documento, en la actualidad las tres partes mencionadas se conservan independientes y sin identificación específica, agrupadas con el resto de documentos musicales del Archivo Diocesano de Menorca bajo la localización genérica $A D M$, partitures. Por este motivo, para describirlas nos referiremos a ellas como bifolios A, B y C.

Los tres pergaminos contienen fragmentos manuscritos de texto con notación musical correspondientes a las Lamentaciones del profeta Jeremías, materiales estrechamente relacionados con las lecturas del primer nocturno de los maitines de Jueves Santo (Feria V), Viernes Santo (Feria VI) y Sábado Santo (Sabbato Sancto). ${ }^{1}$ Mayoritariamente se trata de cantos monódicos codificados en notación cuadrada, excepto un conjunto de versos del capítulo 5 de las Lamentaciones (correspondientes al Sábado Santo) que, aunque también se hallan escritos en notación cuadrada, conforman una sección polifónica a tres voces con añadidos localizados de una cuarta voz en los puntos cadenciales.

Por lo que se refiere a las características físicas y de organización de los documentos, el bifolio B es el que con- serva las medidas más grandes: 510 × $355 \mathrm{~mm}$, de manera que una vez doblado en fascículo originaría folios de 255 x $355 \mathrm{~mm}$. No obstante, éstas no son las originales, ya que en su folio $2 \mathrm{v}$ la inicial de la palabra tenor aparece recortada, lo que induce a pensar que en el reaprovechamiento se debieron recortar ligeramente los márgenes. Pese a ello, la caja de escritura configurada por los tetragramas no se ve afectada, al contrario de lo que sucede en el bifolio C, en el cual se recortó la parte superior de tal manera que del primer tetragrama únicamente se conserva la línea inferior y el texto.

Cada una de las cuatro caras de los bifolios contiene seis tetragramas trazados con tinta roja, distribuidos uniformemente a lo largo de la página con una separación de $25 \mathrm{~mm}$. El texto está escrito con tinta negra, exceptuando algunas iniciales -las que coinciden con el inicio de cada lección-, que son rojas o azules. La indicación de las lecciones y de las voces en la parte polifónica también aparecen escritas con tinta roja.

Si se analiza la correlación del texto que contienen tomando como referencia la secuencia textual de las Lamentaciones que recoge la Vulgata ${ }^{2}$, observamos que el bifolio $\mathrm{C}$ actualmente se conserva doblado al revés de cuando formaba parte del códice -fruto del reaprovechamiento posterior como tapa de libro. Teniendo en cuenta este hecho y efectuando la corrección oportuna, originalmente el bifolio B se hallaría plegado dentro del bifolio A en el mismo fascículo, de manera que los dos serían consecutivos. Por otra parte, el final del folio $1 \mathrm{v}$ del bifolio B no tiene continuidad con el 1r del bifolio $\mathrm{C}$, lo que indica que entre ambos existía otro, por ahora perdido (D), dentro del cual se encontraría el C. De la misma manera, la falta de continuidad entre el final del folio $1 \mathrm{v}$ y el inicio del $2 \mathrm{r}$ del bifolio $\mathrm{C}$ hace suponer que dentro de éste debía existir uno más (E). De este modo, la propuesta de organización de los bifolios y la indicación de su contenido se representa en la figura 1 . 

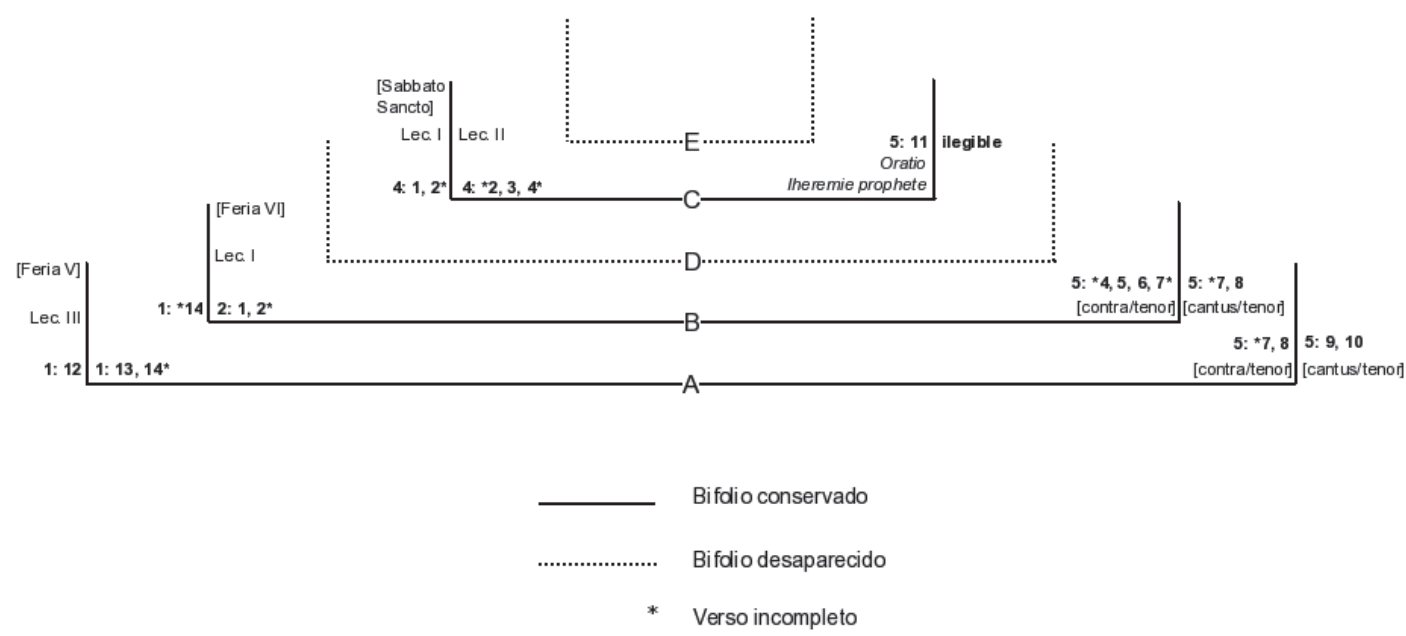

Figura 1. Propuesta de organización de los bifolios y contenido

A partir de la transcripción del texto de los fragmentos conservados y de su ordenación, se obtiene la tabla de la figura 2 , en la cual se representa la procedencia de los textos usados para las tres primera lecciones del primer nocturno del oficio de Jueves Santo (Feria V), Viernes Santo (Feria $V I)$ y Sábado Santo (Sabbato Sancto).

\begin{tabular}{|c|c|c|c|}
\hline & LECTIO & $\begin{array}{l}\text { PROCEDENCIA DEL TEXTO } \\
\text { (capítulo: versos) }\end{array}$ & OBSERVACIONES \\
\hline \multirow{3}{*}{ Feria V } & I & $(?)$ & \multirow{9}{*}{ Tratamiento monódico } \\
\hline & II & $(?)$ & \\
\hline & III & $1: 12,13,14$ & \\
\hline \multirow{3}{*}{ Feria VI } & I & $2: 1,2$ & \\
\hline & II & $(?)$ & \\
\hline & III & $(?)$ & \\
\hline \multirow{4}{*}{ Sabbato Sancto } & $\mathrm{I}$ & $4: 1,2$ & \\
\hline & II & $4: 3,4$ & \\
\hline & \multirow[t]{2}{*}{ III } & 5: (?),11, Oratio Iheremie pphete: & \\
\hline & & $5: 4,5,6,7,8,9,10$ & Tratamiento polifónico \\
\hline
\end{tabular}

Figura 2. Selección de versos utilizados en las Lamentaciones de Jeremías de Ciutadella de Menorca

A partir de la secuencia de bifolios de la figura $1 \mathrm{y}$ del contenido expuesto en la tabla de la figura 2 se puede apreciar que los versos 4-10 del capítulo 5 de las Lamentaciones, que son justamente los que se desarrollan polifónicamente, aparecen después del verso 11 del mismo capítulo, pero en este caso tratado monódicamente y se- guido de la invocación Iherusalem, Iherusalem, convertere ad Dominum Deum tuum, lo que indica la finalización de la lección III. ${ }^{3}$ Este hecho conduce a plantear que en

3 DEL SOL (2010): 254. 
este manuscrito la lección III de Sábado Santo está formada por una selección amplia de versos del capítulo 5 de las Lamentaciones que se cierra con el número 11. Teniendo en cuenta el espacio físico existente entre el final de la lección II y el de la lección III (las cuatro páginas del bifolio E, de acuerdo con la figura 1), y teniendo en cuenta también la configuración de otras Lamentaciones de Jeremías en distintas fuentes del ámbito hispánico ${ }^{4}$, resulta convincente pensar que esta lección III de Sábado Santo estaría formada por los versos 1-11 del capítulo 5. Como el resto de las lecciones anteriores está tratada monódicamente, pero va seguida de una realización polifónica de la misma secuencia de los once primeros versos del capítulo 5 .

Además de las anotaciones más antiguas fruto del reciclaje (inscripción al 31 de desembre 1569 que figura en el bifolio C), la identificación de los versos utilizados en estas Lamentaciones del profeta Jeremías confirma que se trata de una versión pretridentina. La selección del texto, procedente de la Vulgata, no concuerda con el que establece el Breviarium Romanum ${ }^{5}$ promulgado por Pío V el año 1568, mediante el cual se fijó la selección de versos que servirían para las tres primeras lecciones del triduo sacro. ${ }^{6}$

Además, resulta interesante comprobar que el número y la selección de versos utilizados para cada una de las distintas lecciones en el documento de Ciutadella (figura 2) concuerda plenamente con el de tres fuentes de la ciudad de Barcelona: el Breviarium secundum usum ecclessiae Barcinonensis del siglo $\mathrm{XIV}^{7}$, un leccionario conservado en la catedral ${ }^{8}$ y un pasionario impreso en la misma ciudad en 1512. ${ }^{9}$ Según Sergi Zauner, la homogeneidad que presentan estas fuentes, ajustadas a un modelo que se aparta de los usos litúrgicos de otras diócesis vecinas, como Vic o la Seu d'Urgell, y que tampoco coincide con el de otras zonas peninsulares, sugiere la consolidación de una tradición que parece instaurada ya en el siglo XIV y que habría perdurado hasta poco antes del Concilio de Trento.$^{10} \mathrm{La}$ existencia de esta tradición se ve reforzada por la presencia regular, dentro de estas fuentes, de una serie de variaciones textuales en relación a la Vulgata ${ }^{11}$, particularidades del texto que también encontramos en las Lamentaciones de Ciutadella

4 HARDIE (1993): 220-250.

5 Breviarium Romanum (1846): 325-335.

6 HARDIE (1993): 223; DEL SOL (2010): 254.

7 Vic, Arxiu Capitular, Ms 83.

8 Barcelona, Archivo Capitular, Códice 109.

9 Officia trium dierum septimanae sanctae. Barcelona, Carlos Amorós, 1512.

10 ZAUNER (2010): 79-108

11 ZAUNER (2010): 88. de una forma totalmente concordante con las fuentes barcelonesas.

\section{La sección polifónica}

En la parte polifónica las tres voces se distribuyen a lo largo de dos caras enfrentadas: un verso de folio y el recto del folio consecutivo, de manera que los cuatro primeros tetragramas del verso corresponden a la voz más aguda, escrita en clave de do en tercera línea. De esta voz no aparece la denominación, de manera que nos referiremos a ella como cantus. El contra, en clave de fa en tercera, ocupa los cuatro primeros tetragramas del recto del folio consecutivo, mientras que el tenor queda agrupado en la parte baja de ambas caras: los dos últimos tetragramas del verso y los dos últimos del recto siguiente, en este mismo orden de sucesión. La denominación de estas dos voces aparece indicada con tinta roja, fuera de la caja de escritura delimitada por el conjunto de los tetragramas: el tenor en el margen izquierdo y el contra en el derecho. Se puede apreciar claramente esta organización sobre las dos páginas en las imágenes de la figura 3.

Esta distribución de las voces sobre el pergamino se ajusta a la que empiezan a adoptar los motetes a partir de la segunda mitad del siglo XIII, con el triplum y el contra distribuidos en dos columnas y el tenor ubicado en la parte baja. ${ }^{12} \mathrm{Y}$ más concretamente, coincide con la organización que se hace más habitual durante el siglo XV, con las dos voces superiores en dos páginas enfrentadas y el tenor en un único pentagrama en la parte inferior de ambas páginas. ${ }^{13}$ Aunque en los fragmentos que nos ocupan, el tenor se distribuye en los dos últimos tetragramas de cada página, en vez de solamente en el último.

Sin entrar todavía en el análisis musical de los fragmentos, teniendo en cuenta únicamente estas características formales de disposición de las tres voces sobre el pergamino y las fechas que se escribieron en los bifolios cuando posteriormente fueron reutilizados, deberíamos situar el documento dentro del intervalo cronológico que va desde mediados del siglo XIII hasta el último tercio del siglo XVI. En cambio, llama la atención que está escrito en notación cuadrada, cuando precisamente dentro de este período de tiempo se desarrollan las innovaciones más importantes en relación a los aspectos rítmicos de la notación, precisamente para poder codificar de manera satisfactoria el repertorio musical a más de una voz.

12 APEL (1953): 283.

13 PAJARES ALONSO (2011): 55. 

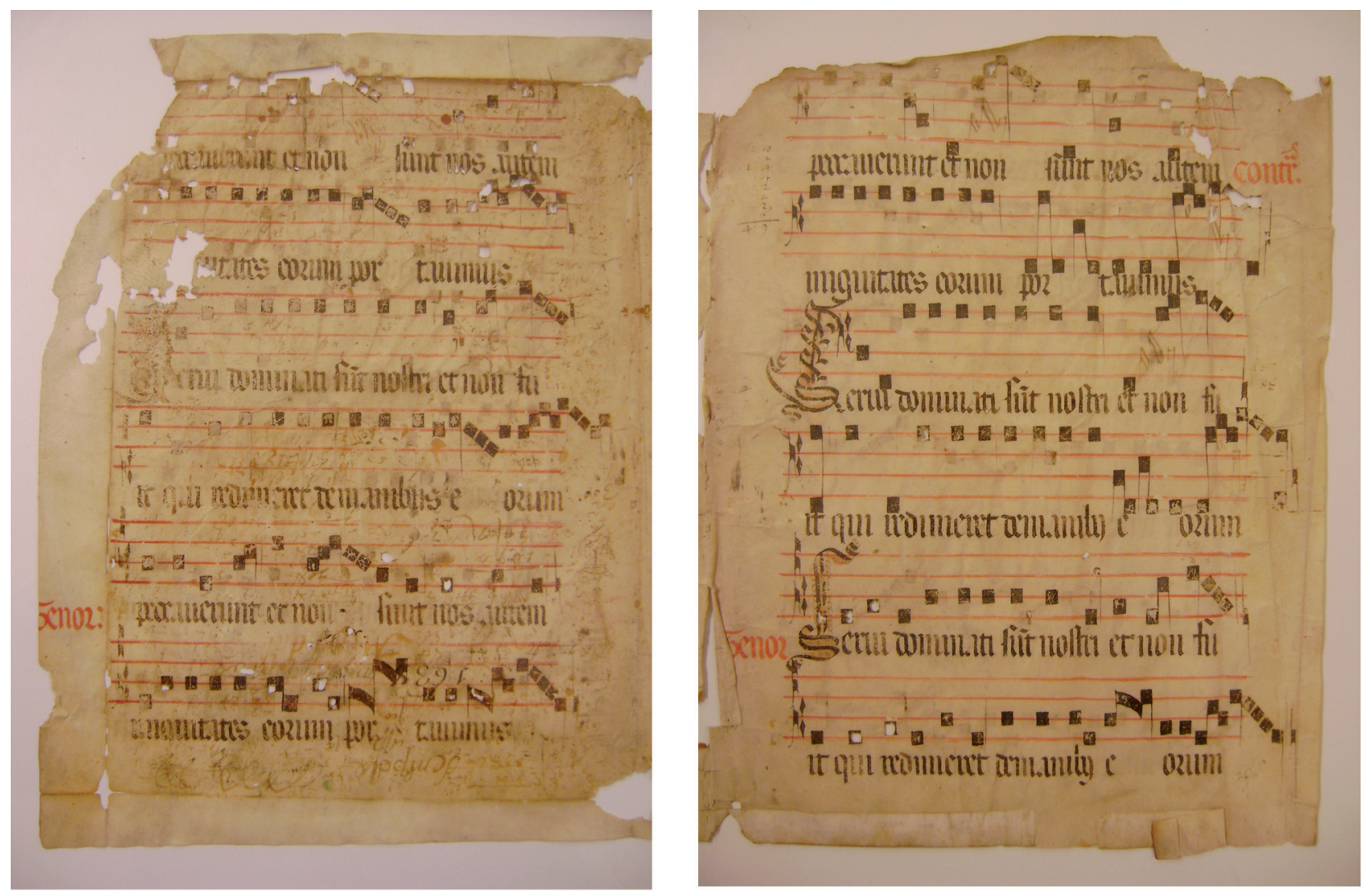

Figura 3. Folio 2v del bifolio B (izquierda), seguido del 2r del bifolio A (derecha), que en la distribución original del fascículo aparecían de manera consecutiva en esta misma disposición para ofrecer las tres partes de la polifonía. Contienen prácticamente todo el verso núm. 7 y la totalidad del número 8 del capítulo 5 de las Lamentaciones

La necesidad de crear códigos escritos adecuados para indicar la superposición precisa de las diferentes líneas melódicas de las obras polifónicas fue el motivo principal que empujó al desarrollo de la notación para conseguir sistemas cada vez más exactos rítmicamente hablando, comenzando por los modos rítmicos del siglo XIII hasta la notación blanca mensural del siglo XVI.

Todo esto nos lleva a afirmar que en la sección polifónica objeto de este estudio el ritmo no era un aspecto de gran relevancia o, como mínimo, no ofrecía una especial complicación. Confirmaría esta hipótesis el hecho de que, exceptuando momentos muy puntuales, toda la polifonía es de tipo nota contra nota, incluso en las partes melismáticas.

De esta sección polifónica hemos podido reconstruir íntegramente los versos 7 y 8 del capítulo 5, exceptuando las cuatro primeras notas del cantus del primero. Del verso núm. 6 se conservan las partes del contra y del tenor-falta el cantus-, mientras que en el verso 9 falta la parte del contra. Desde la palabra final del verso 4 (comparavimus) hasta el final del verso 5 se conserva únicamente el contra, mientras que del verso 10 únicamente tenemos el cantus.

En la figura 4 se ofrece la transcripción de los versos 6-9, los que conservan al menos dos de las tres voces. Los criterios utilizados han sido los siguientes:

- Teniendo en cuenta que las Lamentaciones están codificadas en notación cuadrada, se han transcrito únicamente los perfiles melódicos de cada voz, sin proponer figuración rítmica.

- Los neumas se han mantenido indicados mediante ligaduras que agrupan las notas que los componen.

- Las barras verticales continuas indican el final de cada verso del texto.

- Las barras verticales discontinuas se corresponden con los signos de separación del texto (las peque- 
ñas líneas verticales que aparecen a lo largo de los tetragramas).

- La relación del texto con la notación del manuscrito ha servido de base para la transcripción, aunque se ha revisado a fin de conseguir una distribución lógica de las sílabas en relación con los neumas y las notas repetidas.
- Cada sistema equivale a un verso, indicado numéricamente entre corchetes en la parte superior [capítulo: verso].

- El texto se ha mantenido con las particularidades ortográficas del manuscrito, mientras que las mayúsculas y la puntuación se han ajustado al texto de la Vulgata.

Oracio Iheremie prophete

[Capitulo 5]
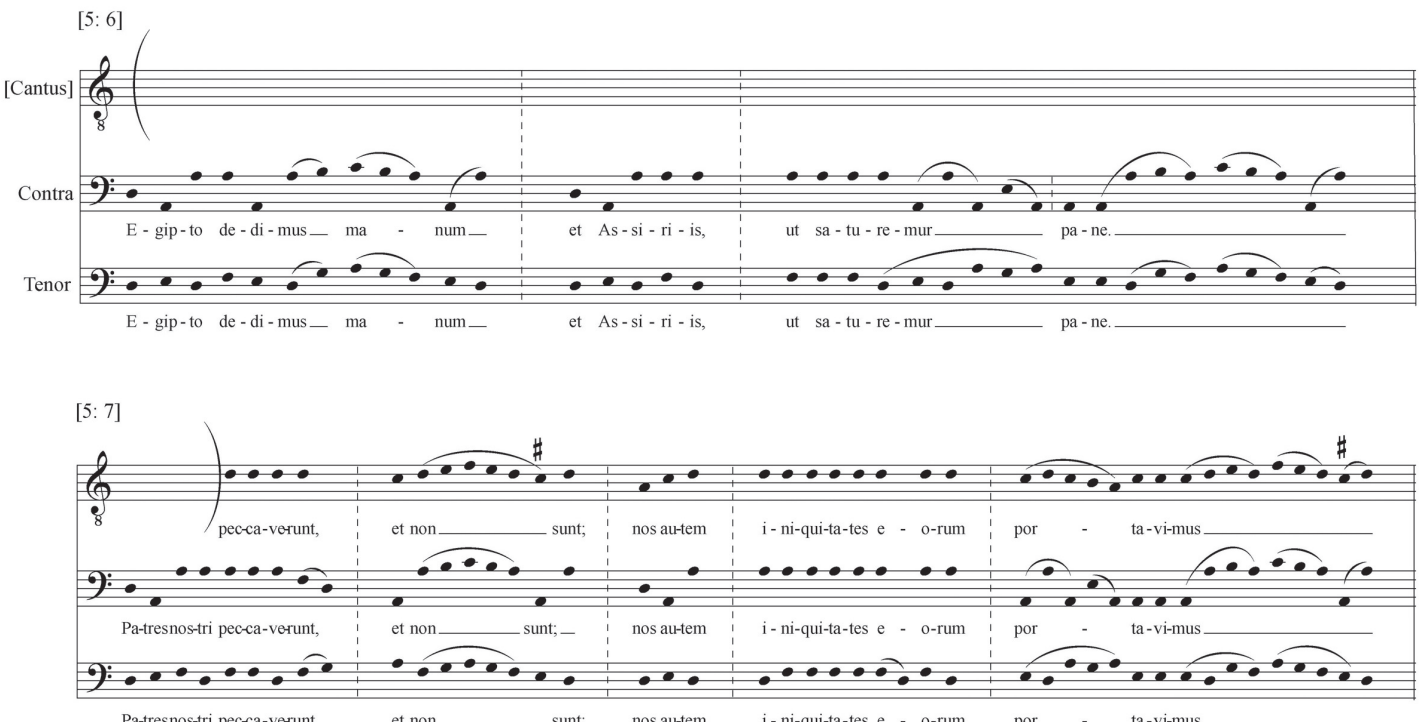

Pa-tresnos-tri pec-ca-verunt, et non__ sunt; nos au-tem i-ni-qui-ta-tes e - o-rum por $\quad$ - ta-vi-mus

[5: 8$]$

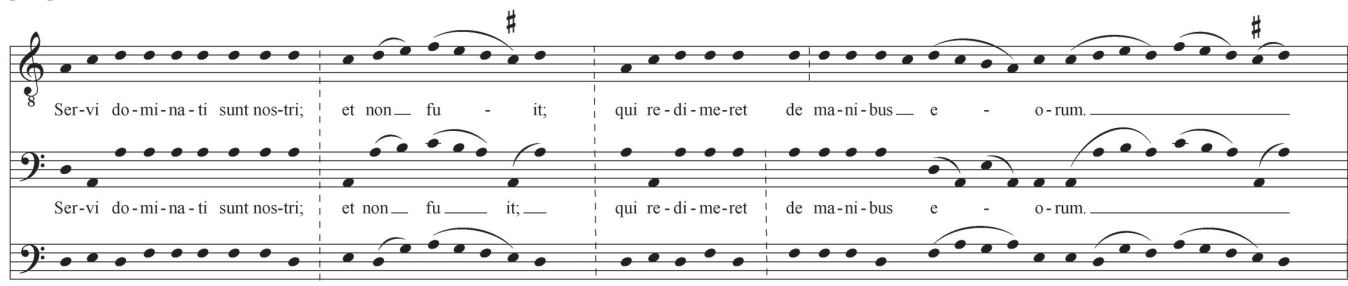

Ser-vi do-mi-na-ti sunt nos-tri; et non_ fu__ it; qui re-di-me-ret de ma-ni-bus e - o-rum.

[5:9]

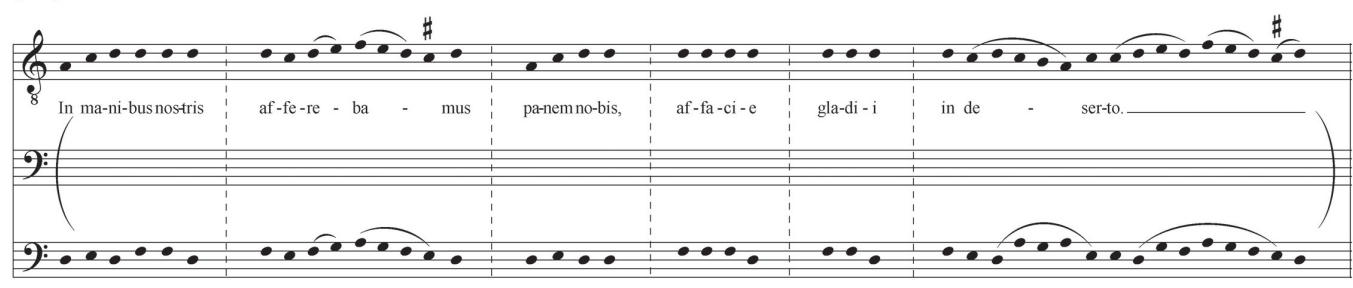

In ma-ni-busnostris af-fe-re - ba - mus pa-nemno-bis, af-fa-ci-e gla-di-i $\quad$ in de-ser $\quad$ to.

Figura 4. Transcripción de la realización polifónica de los versos 6-9 del capítulo 5 de les Lamentaciones de Jeremías del Archivo Diocesano de Menorca 


\section{Organización musical de la polifonía}

De los versos tratados polifónicamente en estas Lamentaciones únicamente se conservan completos los números 7 y 8 , mientras que a los restantes les faltan una o dos voces. No obstante, el material es suficiente para poder describir y reconstruir la organización de este tipo de polivocalidad.
Los siete versos se suceden consecutivamente siguiendo el orden literario de la Vulgata, aunque con algunas diferencias que se indican en la figura 5. Cada uno se desarrolla sobre un recorrido musical que se expone tantas veces como versos forman el conjunto, a manera de estrofa, de manera que se establece un funcionamiento de tipo cíclico. Pero las sucesivas exposiciones tienen extensiones diferentes, debido al número variable de sílabas de cada verso.

\begin{tabular}{|l|l|}
\hline VULGATA & MANUSCRITO DEL ADM \\
\hline 4 & 4 \\
$\begin{array}{l}\text { Aquam nostram pecunia bibimus; } \\
\text { Ligna nostra pretio comparavimus. }\end{array}$ & {$[\ldots]$} \\
5 & 5 \\
Cervicibus nostris minabamur, & Cervicibus minabatur, \\
Lassis non dabatur requies. & Lassis non dabatur re[quies]. \\
6 & 6 \\
Aegypto dedimus manum et Assyriis, & Egipto dedimus manum et Assiriis, \\
Ut saturaremur pane. & Ut saturemur pane. \\
7 & 7 \\
Patres nostri peccaverunt, et non sunt; & Patres nostri peccaverunt, et non sunt; \\
Et nos iniquitates eorum portavimus. & Nos autem iniquitates eorum portavimus. \\
8 & 8 \\
Servi dominati sunt nostri; & Servi dominati sunt nostri; \\
Non fuit qui redimeret de manu eorum. & Et non fuit qui redimeret de manibus eorum. \\
9 & 9 \\
In animabus nostris afferebamus panem nobis, & In manibus nostris afferebamus panem nobis, \\
A facie gladii in deserto. & A facie gladii in deserto. \\
10 & 10 \\
Pellis nostra quasi clibanus exusta est, & Pellis nostra quasi clibanus exusta est, \\
A facie tempestatum famis. & {$[$ A] facie tempestatis et famis. } \\
& \\
\hline
\end{tabular}

Figura 5. Tabla comparativa del texto del capítulo 5 de las Lamentaciones usado en el manuscrito de Ciutadella de Menorca y el que establece la Vulgata. ${ }^{14}$ Las diferencias en el texto se han marcado en negrita 
Para entender la construcción de esta polifonía es necesario partir de la melodía utilizada en las Lamentaciones monódicas precedentes, los fragmentos conservados pertenecientes al Jueves Santo, al Viernes Santo y al Sábado Santo. Estos versos se desarrollan sobre una melodía que se ajusta al tonus lamentationum hispano -tomando la denominación utilizada por Manuel Del Sol-, una configuración melódica característica de las Lamentaciones del ámbito hispánico que podría estar relacionada con estructuras melódi- cas propias de la liturgia hispánica anterior a la implantación del repertorio gregoriano. ${ }^{15}$ En el caso de las Lamentaciones halladas en Ciutadella, esta melodía se identifica por la secuencia inicial de grados La-Do-Re, con el Re como primera cuerda de recitación, seguida de la secuencia Re-Fa-Sol, con el Sol como segunda cuerda de recitación. En algunos casos, como en la lección II del Viernes Santo (figura 6) aparece hasta un tercer recitado, éste sobre el grado La.

Feria VI, lectio II [2:1]

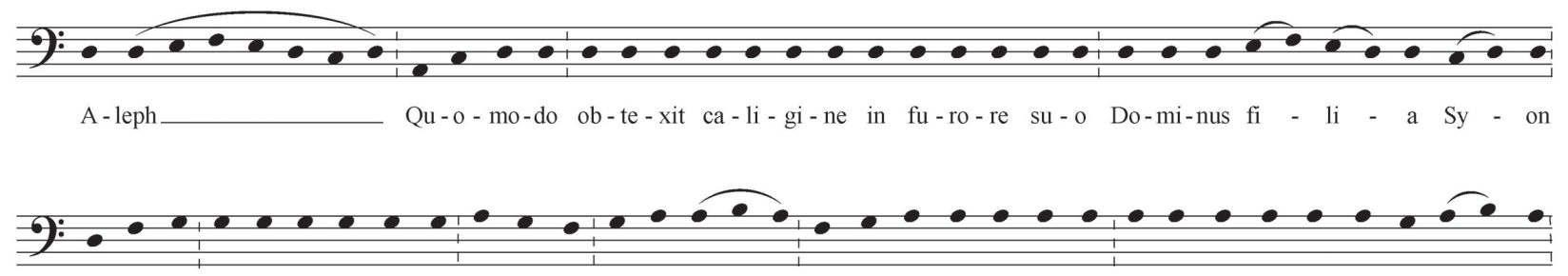

pro-ie - cit de ce-lo in ter-ram in - cli-tam Isr-ra-el et non re-cor-da-tus est sca - bel-lum pe-dum su-o - rum

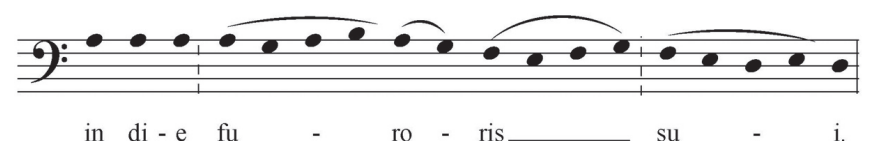

Figura 6. Lección II del Viernes Santo de las Lamentaciones de Jeremías de Ciutadella de Menorca

En los versos polifónicos conservados en este conjunto de Lamentaciones se utiliza la primera parte de la melodía de las lecciones monódicas. Concretamente, la célula inicial La-Do-Re, con el Re como único grado para la recitación. Esta línea melódica se ubica en la parte más aguda de la polifonía, es decir, en el cantus. Cada verso, que como ya hemos explicado equivale a una unidad musical, se divide en dos segmentos desiguales, el primero más corto que el segundo (figura 7). Ambos se basan en la misma secuencia melódica, de manera que el inicio y la terminación se mantienen, al igual que la cuerda de recitación, pero el segundo segmento se presenta más desplegado y ornamentado. Esta correspondencia se aprecia más claramente en la figura 8 , en la cual los dos segmentos que forman la melodía del cantus de un verso completo se han dispuesto de manera sinóptica, alineando verticalmente aquellos elementos equivalentes. ${ }^{16}$ 
segmento $1^{\circ} \quad$ segmento $2^{\circ}$

[Cantus]

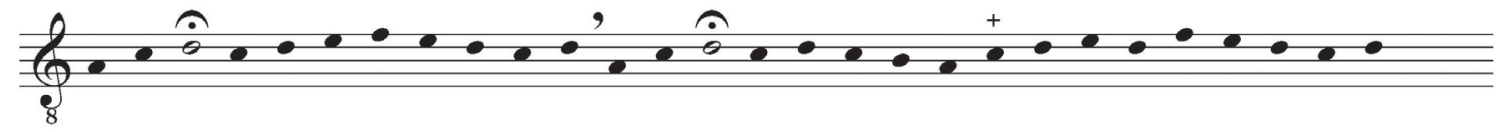

Figura 7. Secuencia melódica del cantus correspondiente a un verso completo. El calderón identifica el grado de la cuerda de recitación $(\mathrm{Re})$, mientras que la cruz indica que en este punto el grado Do se reitera un número variable de veces

en función de las sílabas del texto

seg. $1^{\circ}$

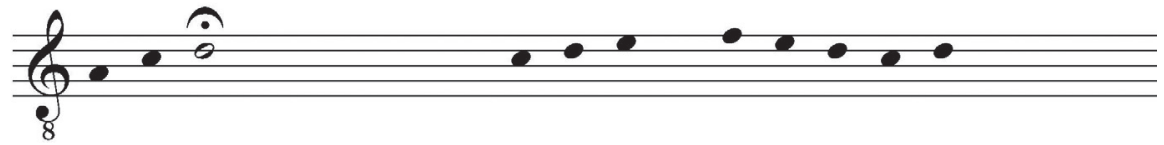

$\operatorname{seg} .2^{\circ}$

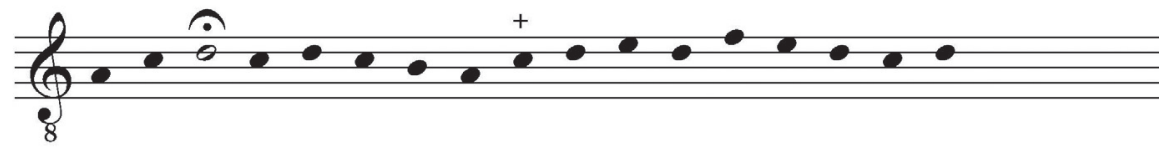

Figura 8. Disposición sinóptica de la secuencia melódica del cantus. Se puede observar que el segundo segmento es una reformulación más desplegada del primero, pero manteniendo la fórmula de inicio y la de terminación

Pero la correspondencia dentro los dos segmentos melódicos de cada unidad no es exclusiva de la voz del cantus, sino que también tiene su reflejo en las otras dos partes (contra y tenor), según se muestra en la figura 9.

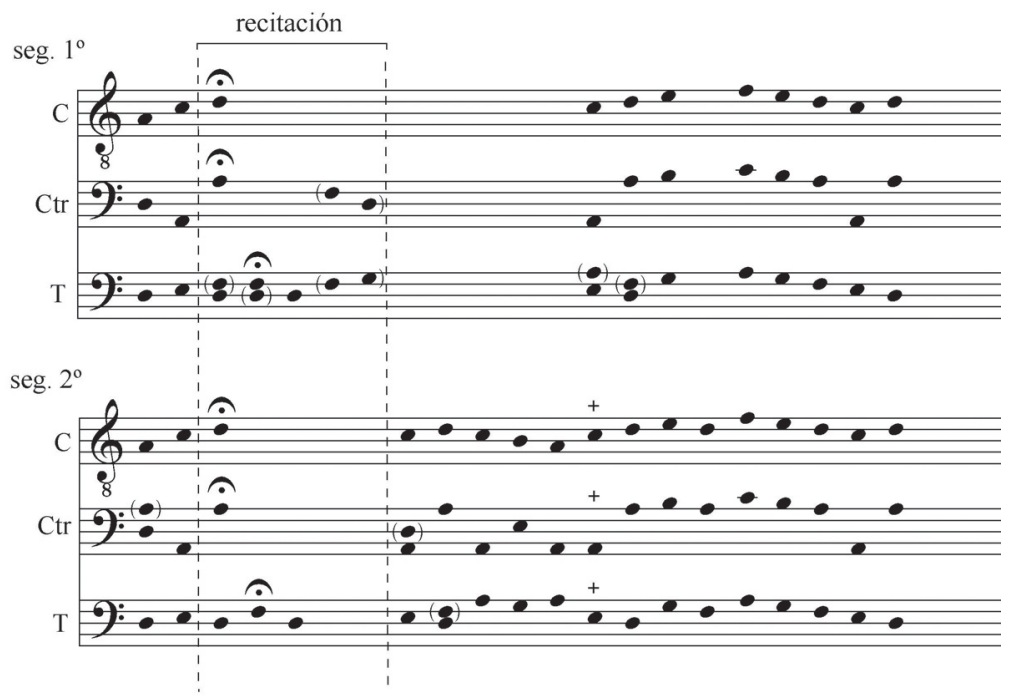

Figura 9. Disposición sinóptica del conjunto de las tres voces de un verso. Las notas que figuran entre paréntesis son variantes observadas a lo largo de los demás versos conservados 
Resulta interesante observar que la compartimentación de la unidad musical en dos segmentos no siempre concuerda con la división del verso en dos hemistiquios. De los cuatro versos transcritos en la figura 4 , solamente en el número 7 coinciden los dos sistemas de división, como se puede apreciar en la figura 10. Por lo tanto, este canto a tres voces se puede entender como el resultado de aplicar un pro- cedimiento recurrente a un texto sin tener del todo en cuenta su organización literaria. Este hecho refuerza la hipótesis de que este tipo de polifonía, que como veremos posteriormente se ajusta a un fabordón, podría tener su origen en un tipo de polivocalidad improvisada a partir de una melodía dada, en contraposición a la composición polifónica escrita. ${ }^{17}$

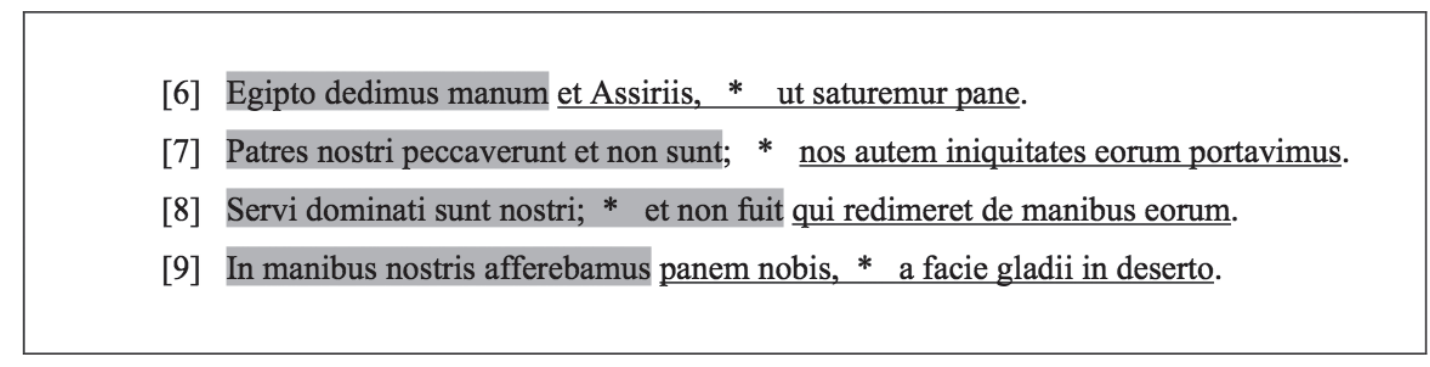

Figura 10. Relación entre la estructura del texto y la segmentación musical. El texto sombreado corresponde al primer segmento musical de cada unidad, mientras que subrayado forma parte del segundo segmento.

El asterisco indica la separación entre los dos hemistiquios del verso

En cuanto a la relación entre el texto y la melodía, en general predomina el tratamiento silábico, excepto en los finales de cada segmento. De este modo, los inicios -la secuencia melódica La-Do-Re- y el recitado sobre Re son estrictamente silábicos. En cambio, los perfiles melódicas que aparecen a continuación de los recitados en ambos segmentos quedan configurados como melismas que conducen a la cadencia. En el primer segmento el giro Re-Mi corresponde a una misma sílaba y la secuencia siguiente (Fa-Mi-Re-Do), a otra. En el segundo segmento, las secuencias Re-Do-SiLa-Do y Do-Re-Mi-Re-Fa-Mi-Re-Do-Re constituyen dos melismas de 5 y 9 notas respectivamente.

Pese a las diferentes extensiones de los versos originadas por el número variable de sílabas, el segmento literario se ajusta siempre al mismo esquema melódico expuesto. Esto es posible gracias a la flexibilidad de la cuerda de recitación, que admite más o menos texto variando simplemente el número de veces que se reitera el grado $\mathrm{Re}$, mientras que el inicio y la terminación de cada segmento se mantienen siempre regulares. Este proceso también se observa, aunque en menor medida, sobre el grado Do del segundo segmento (indicado mediante una cruz en las figuras 7, 8 y 9). Este procedimiento elástico que permite alargar o acortar la extensión del modelo gracias a la reiteración -más o menos veces- de partículas interiores mientras los inicios y los finales de los segmentos se mantienen invariables también se observa en músicas instrumentales de transmisión oral de diferentes áreas del Mediterráneo: es el caso de algunas músicas de danza de la zona central de Italia o buena parte del repertorio litúrgico y también el de danza para flaüta y tambor de las islas de Eivissa y Formentera. ${ }^{18}$ Se trata de un mismo principio mecánico que permite adaptar la estructura sonora al texto en el caso de las Lamentaciones, o bien a las acciones a las que acompaña la música -el desarrollo de la liturgia o las danzas- en el caso de las músicas instrumentales.

A partir de la melodía del cantus se añaden dos líneas melódicas más por la parte inferior, el contra y el tenor, que originan una polifonía de nota contra nota que únicamente se rompe en tres momentos muy concretos. Estas excepciones son la última sílaba del texto peccaverunt y la primera sílaba del texto eorum (figura 4, verso 7), con dos notas de las voces inferiores contra una sola del cantus; y la última sílaba de manibus (figura 4, verso 8), en donde sucede lo contrario: aparecen dos notas de la voz superior para una sola del contra y del tenor.

El tenor tiene un comportamiento melódico más similar al del cantus, con predominio de los grados conjuntos, diversos saltos de $3^{\mathrm{a}}, 4^{\mathrm{a}}$ y uno de $5^{\mathrm{a}}$ como intervalo melódico más grande. En cambio, el contra se caracteriza por una presencia importante de saltos de $8^{\text {a }}$, concretamente entre los sonidos $\mathrm{La}_{1}$ y $\mathrm{La}_{2}$. Aunque durante la mayor parte del verso esta voz se desarrolla dentro del ámbito sonoro $\mathrm{La}_{2}-\mathrm{Do}_{3}$-por encima del tenor-, los saltos constantes al $\mathrm{La}_{1}-\mathrm{y}$ en menor medida, al $\mathrm{Re}_{2}$ y al $\mathrm{Mi}_{2}-$ implican que en este punto sea el contra la voz más

17 MACCHIARELLA (1995): 140. 18 GIURIATI (1987): 251-253; ESCANDELL (2010): 225-250. 
grave, de manera que se producen cruzamientos constantes entre las líneas melódicas del tenor y del contra.

Las secuencias melódicas del contra y del tenor en muchos momentos se desarrollan de manera paralela a la melodía del cantus. Este comportamiento paralelo aparece en los recitados y en la parte final de los melismas cadenciales de cada segmento. Estas áreas, que sumadas suponen prácticamente la mitad de la extensión del verso, presentan una disposición en la cual el contra se encuentra una $4^{\mathrm{a}}$ justa por debajo del cantus y el tenor una $3^{\text {a }}$ mayor o menor por debajo del contra. Tal disposición, analizada desde una perspectiva armónica, equivaldría a una configuración de acorde tríada en primera inversión.

En el inicio y la terminación de cada segmento se produce una sonoridad de $5^{\mathrm{a}}$ justa con el $\mathrm{La}_{2}$ del contra sobre el $\operatorname{Re}_{2}$ del tenor, el cual se duplica en la octava superior en el cantus. Esta disposición vertical aparece numerosas veces a lo largo del verso, siempre sobre el Re del tenor, grado que funciona como centro tonal de la pieza.

También aparecen, aunque en menor medida, disposiciones armónicas de tríada en estado fundamental, que en algunos casos les falta la $5^{\mathrm{a}}$ justa. Se presentan principalmente sobre el La ${ }_{1}$ del contra (cruzado por debajo del tenor), con el $\mathrm{Mi}_{2}$ en el tenor y el $\mathrm{Do}_{3}$ en el cantus.

La proporción de las tres tipologías de sonoridades descritas, calculada a partir del verso 8 , es la siguiente: $45 \%$ de paralelismos estrictos de $4^{\mathrm{a}}$ y de $6^{\mathrm{a}}$ inferiores a partir del cantus; $30 \%$ de consonancias perfectas de $5^{\mathrm{a}}$ y $8^{\mathrm{a}}$; y $25 \%$ de disposiciones triádicas en estado fundamental (con posible ausencia de la $5^{\text {a }}$ ). La distribución de estas sonoridades queda representada en la figura 11.

[5: 8]

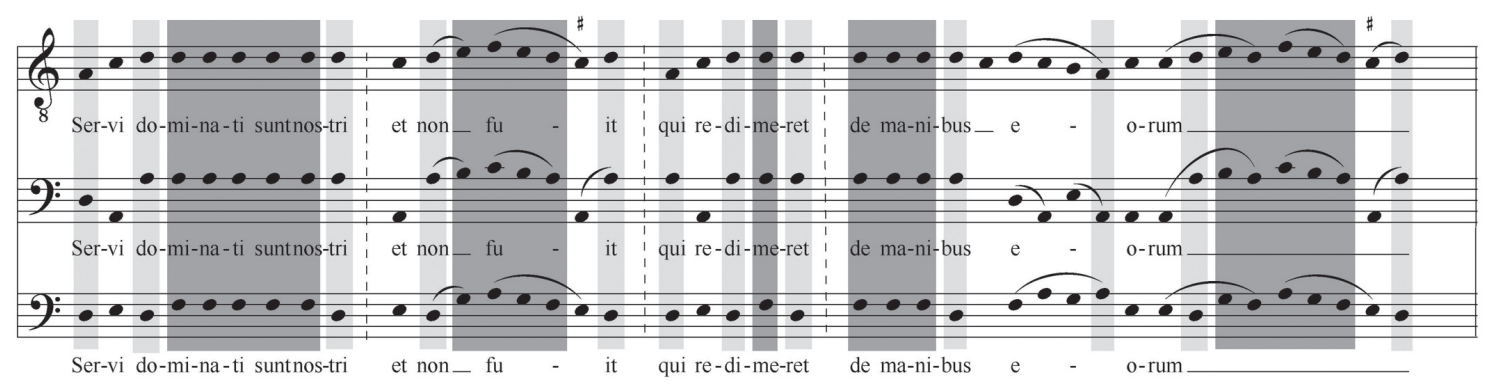

Disposición de tríada en primera inversión ( $4^{\mathrm{a}}$ y $6^{\mathrm{a}}$ inferiores a partir del cantus $)$

Consonancias de $8^{\mathrm{a}}$ y $5^{\mathrm{a}}$ justas

Tríadas en estado fundamental

Figura 11. Distribución de los diferentes tipos de sonoridades simultáneas a lo largo del verso 8 del capítulo 5

La configuración de las voces que acabamos de describir permite afirmar que las Lamentaciones de Jeremías de Ciutadella de Menorca se ajustan a las características de los ejemplos más tempranos de fabordones de la Europa continental, fechables a mediados del siglo $\mathrm{XV}^{19}$, con la particularidad que en estas Lamentaciones las tres voces aparecen desplegadas por escrito.

19 TROWELL (2001): vol VIII, 614-620.
Pero además de estas tres voces que se copiaron inicialmente, en los tetragramas correspondientes al contra aparecen una serie de figuras añadidas en puntos específicos del desarrollo de los segmentos melódicos que conforman cada verso. Concretamente, se encuentran siempre en el último melisma de cada segmento, en forma de notación cuadrada que solamente presenta el contorno de las figuras, sin ennegrecido interior, de manera que morfológicamente adopta la apariencia de la notación blanca del siglo XV (véase la figura 12). 

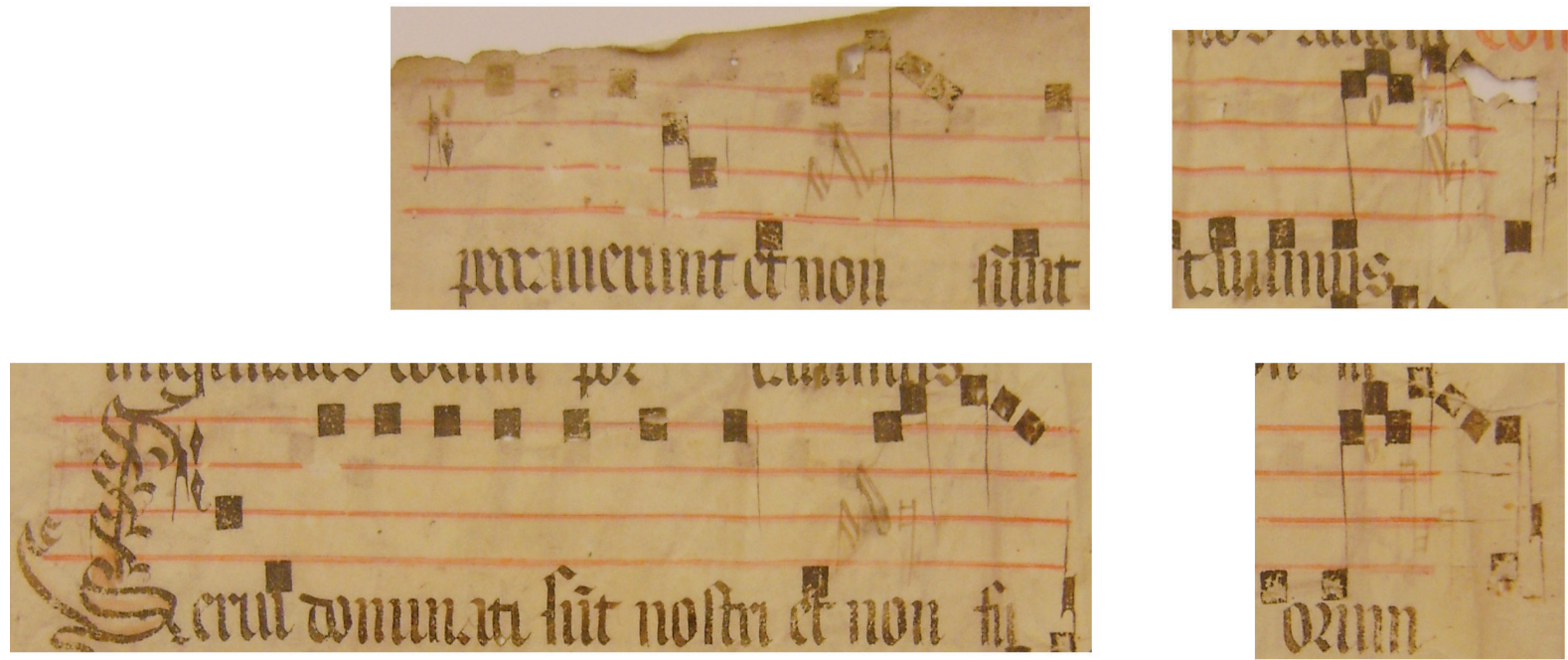

Figura 12. Detalles de las figuras añadidas en los tetragramas del contra: versos 7 (arriba) y 8 (abajo) del capítulo 5 .

Se puede apreciar la recurrencia de los diseños utilizados

En general, la grafía de estas figuras es más pequeña, irregular y mucho menos cuidada que la de la notación cuadrada original, y debe entenderse como un añadido posterior que podría constituir una cuarta voz o bien una línea melódica alternativa a la del contra en estos puntos concretos, aunque no se aprecian signos de raspado en la notación original.

Al final del primer segmento melódico del verso la notación añadida responde siempre a la secuencia Re-Do-FaDo-Re, mientras que al final del segundo la fórmula es SolFa-Do-Re. La dificultad radica en cómo encajar estas series de sonidos con el restos de las voces, teniendo en cuenta el contexto no mensural. Si se contemplan las cuatro voces, las diferentes posibilidades de distribución (nota contra nota o bien alargando alguno de los sonidos de la voz añadida con- tra dos o tres del resto de las voces) originan siempre, en un momento u o otro, intervalos de $2^{\mathrm{a}}$ o de $7^{\mathrm{a}}$ que resultan más bien extraños en este tipo de lenguaje. En cambio, si estas secuencias se consideran realmente una alternativa a la notación original del contra, de manera que al final del primer segmento se sustituye la secuencia original La-Si-Do-Si-La por la de la notación añadida Re-Do-Fa-Do-Re, y al final del segundo segmento, en el neuma La-La-Si-La se sustituye el Si por un Sol (respetando la posición específica en la cual aparece siempre esta figura añadida) y el neuma siguiente Do-Si-La se sustituye por Fa-Do-Re (al igual que sucede en este mismo giro melódico al final del primer segmento), se obtiene la solución representada en la figura 13.

\section{[5:8]}

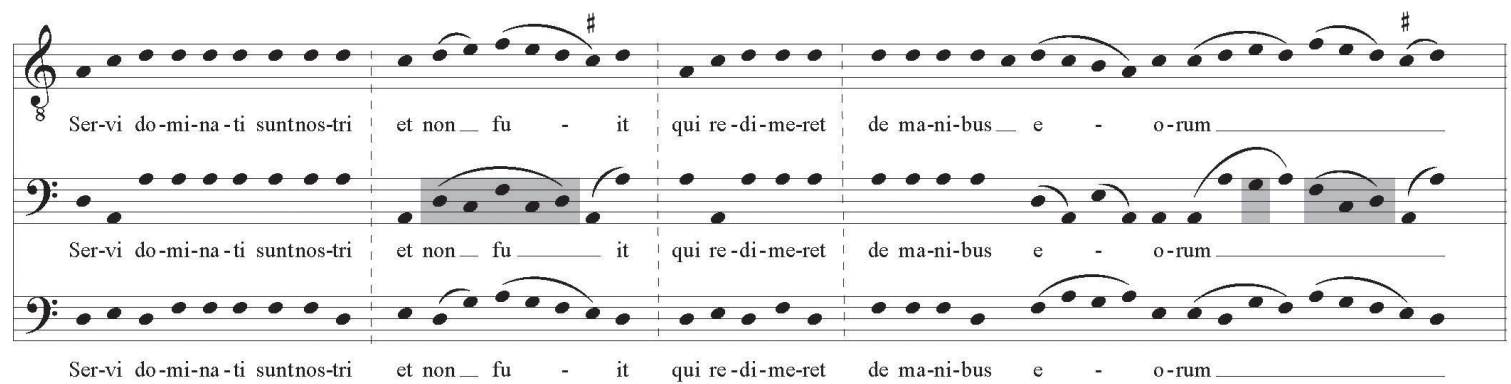

Figura 13. Verso 8 del capítulo 5 de las Lamentaciones, con las secuencias alternativas de la voz del contra remarcadas mediante sombreado 
Si las notaciones añadidas se consideran realmente alternativas puntuales a la voz original del contra tal como hemos explicado en el párrafo anterior, las consecuencias a nivel sonoro son relevantes: los melismas finales de cada segmento musical, que en la figura 11 funcionan como paralelismos de acordes en primera inversión -con una sonoridad caracterizada por las cuartas paralelas entre el cantus y el contra-, en la figura 13 el contra cruza por debajo del tenor y se origina una serie de acordes en estado fundamental, en los cuales el contra ya no se mueve paralelamente con las otras dos voces y los paralelismos se reducen a las sextas entre el cantus y el tenor, de manera que desaparecen las cuartas paralelas que proporcionaban la sonoridad más arcaica de la versión original.

Este hecho reforzaría la hipótesis de que las notaciones añadidas constituyen una serie de correcciones efectuadas con posterioridad a la fijación por escrito de las tres voces originales con la finalidad de sustituir unas sonoridades que posiblemente ya se consideraban anticuadas -los paralelismos de cuartas justas del fabordón inicial- por otras más contemporáneas, basadas en una estructura de tríadas en estado fundamental. Aunque de momento tenemos demasiada poca información para poder datar con precisión, nos podemos aventurar a proponer que estos cambios se podrían haber efectuado hacia las últimas décadas del siglo XV o durante los primeros años del siglo XVI, mientras que el fabordón original podría situarse durante la primera mitad o parte central del siglo XV. No obstante, estos márgenes temporales tan amplios, establecidos por el momento en base al tipo de lenguaje sonoro, deberán acotarse a partir de futuros estudios de los registros documentales de los centros de procedencia de los documentos, como inventarios o libros de cuentas que puedan proporcionar algún tipo de referencia sobre manuscrito.

Como ya hemos dicho anteriormente, los bifolios se conservan en el Archivo Diocesano de Menorca, pero forman parte de un fondo procedente del convento de Els Socors de Ciutadella, perteneciente a la orden de San Agustín. La cronología de los bifolios es anterior al actual convento, levantado durante el siglo XVII. ${ }^{20}$ En todo caso, se podrían relacionar con el antiguo monasterio que los frailes agustinos tenían en el puerto de Ciutadella. De dicho edificio existen referencias documentales del año $1480^{21}$, y debido a su emplazamiento -en la parte exterior del perímetro de las murallas- fue severamente devastado en el asalto turco de 1558 , por lo que se supone que buena parte de los religiosos mu-

20 VILLALONGA y FIOL (2009): 5-18.

21 CASASNOVAS y SASTRE (2010): 46; SASTRE (1982): 15 i 21 . rieron en aquel ataque. En 1562 volvió a ser habitado, pero se abandonó nuevamente en 1573, cuando los religiosos se integraron en el convento de Els Socors de Palma (Mallorca). Tres años después, en 1576, la orden se instala de nuevo en Ciutadella de Menorca. ${ }^{22}$

Teniendo en cuenta el alto grado de destrucción que seguramente padeció el convento de extramuros de la orden de San Agustín durante el ataque de 1558 -hasta el punto de ser abandonado-, difícilmente se puede considerar que los fragmentos musicales estudiados procedan de ese monasterio. En cambio, resulta más factible pensar que los bifolios podrían haber llegado ya a Menorca reaprovechados como tapas de otros libros, procedentes del mismo convento de Els Socors de Palma, cuando los religiosos agustinos regresaron nuevamente a Ciutadella de Menorca, a partir de 1576. Pero todas estas posibilidades, que de momento son meras conjeturas, deberán ser validadas o rebatidas a partir de futuras investigaciones.

\section{Conclusiones}

Las ideas principales que podemos extraer de esta primera aproximación a los tres bifolios localizados en el Archivo Diocesano de Menorca, y más concretamente del análisis de la sección polifónica que contienen, quedan resumidos en los siguientes puntos:

1. La parte final de las Lamentaciones del profeta Jeremías localizadas en el ADM constituyen un tipo de polifonía que se desarrolla a partir de la melodía utilizada en las Lamentaciones monódicas que preceden la parte polifónica. A dicha melodía, situada en el cantus, se le añaden dos líneas melódicas por debajo - un contra y un tenor- que se mueven principalmente de manera paralela al cantus, a distancia de $4^{\mathrm{a}}$ y de $6^{\mathrm{a}}$ inferiores en relación a la voz superior, excepto en los inicios y en las cadencias de los segmentos que forman cada verso, en donde se utilizan sonoridades de $5^{\mathrm{a}}$ y $8^{\mathrm{a}}$ justas. Se trata, por tanto, de un procedimiento polivocal que podemos clasificar como fabordón.

2. La aplicación de un mismo esquema musical a los distintos versos de las Lamentaciones que se ajusta a las extensiones variables del texto, sumado a la particularidad de que no concuerda la organización musical y la literaria, conducen a pensar que este fabordón refleja una práctica eminentemente

22 JULIÀ (1988): 4 i 5; VILLALONGA y FIOL (2009). 
de transmisión oral que implicaría cierto grado de improvisación: una técnica para embellecer el canto monódico -en este caso, situado en el cantus- que en un determinado momento se habría fijado por escrito.

3. Esta parte polifónica que aparece al final de las Lamentaciones constituye una realización polivocal de los mismos versos 1-11 que inmediatamente antes se habían expuesto monódicamente como material para la lección III del Sábado Santo. Por tanto, podemos hablar de una versión más ornamentada que no queda demasiado claro si complementaría o sustituiría la parte monódica correspondiente. Existe la posibilidad de que esta realización polivocal, aunque no se trate de una polifonía muy elaborada, pudiese tener un uso más flexible dentro de la liturgia, sin que necesariamente tuviese que ocupar la ubicación que le correspondería en las lecturas de Tinieblas, tal como algunos autores sugieren para otras Lamentaciones polifónicas del ámbito hispánico. ${ }^{23}$

4. El comportamiento del contra, con una presencia importante de saltos de octava y cruzamientos constantes con el tenor, se ajusta al que adopta esta misma voz en composiciones vocales a tres voces de la parte central del siglo XV. La propia configuración original a tres voces, sumada al procedimiento del fabordón y a las cadencias con consonancia de $5^{\mathrm{a}}$ justa y ausencia de $3^{\mathrm{a}}$, concuerdan con el lenguaje sonoro de la época. Todas estas características técnicas y estilísticas conducen a pensar que la sección polifónica de las Lamentaciones que aquí tratamos puede situarse cronológicamente dentro del siglo $\mathrm{XV}$, preferentemente en su parte central o, incluso, durante la primera mitad. De confirmarse esta hipótesis, nos encontraríamos ante uno de los ejemplares más antiguos de Lamentaciones polifónicas del ámbito hispánico ${ }^{24}$, aunque en este caso se trate de un tipo de polivocalidad relativamente simple, de autoría anónima y con rasgos más propios de prácticas de transmisión oral, de manera que el resultado se diferencia de las composiciones polifónicas más elaboradas de autores conocidos. ${ }^{25}$

5. La presencia de las notaciones alternativas en la voz del contra al final de los segmentos musicales, con una grafía mucho más sencilla y poco cuidada en relación a la que presentan las tres voces originales, se podría interpretar como correcciones derivadas de cambios estéticos y de concepción sonora. Cambios que pasarían por transformar las sonoridades iniciales del fabordón (con paralelismos de cuartas y sextas), en una serie de acordes tríadas en estado fundamental, con el refuerzo de la concepción acordal que este hecho implica, de manera que estas correcciones podrían situarse hacia el final del siglo XV o a principios del XVI.

6. La concordancia entre el texto de las Lamentaciones conservadas en Ciutadella y el de otras Lamentaciones contenidas en fuentes barcelonesas de los siglos XIV, XV y principio del XVI por lo que se refiere a la selección de los versos utilizados en cada una de las lecciones (figura 2) y a las particularidades que adopta el texto en relación a la versión de la Vulgata, indica una conexión entre ambos lugares, relación fácilmente argumentable teniendo en cuenta que, a nivel de organización política, la isla de Menorca formaba parte de la Corona de Aragón desde 1287, cuando fue conquistada a los musulmanes por el rey Alfonso III de Aragón.

7. La procedencia exacta de los bifolios es, por ahora, el aspecto más incierto. Si partimos del hecho que el mismo ADM considera que forman parte de un fondo que proviene del convento de Els Socors de Ciutadella -de la orden de San Agustín-, lo más convincente es que estos documentos ya hubiesen llegado a Menorca reaprovechados como tapas de otros libros, traídos por los religiosos cuando regresaron para instalarse de nuevo en la isla procedentes del convento de Els Socors de Palma, en 1576, con posterioridad a que el ataque turco que devastó Ciutadella de Menorca ocasionase la destrucción del antiguo monasterio que la orden de San Agustín tenía extramuros. No obstante, otras hipótesis son posibles, desde considerar que los documentos se hubiesen podido salvar de la destrucción del antiguo convento en 1558, o que llegasen a Menorca desde otros puntos peninsulares como Barcelona -a juzgar por las concordancias con el texto de otras Lamentaciones de esta ciudad- o Valencia, provincia eclesiástica a la cual perteneció el antiguo convento de los agustinos de Ciutadella hasta $1569 .{ }^{26}$ En cualquier caso, se trata de aspectos que necesariamente deberán ser objeto 
de futuras investigaciones para poder sacar a la luz más información que permita una datación mucho más precisa de los documentos que aquí hemos presentado.

\section{Bibliografía}

Apel, Willi, The notation of polyphonic music 900-1600. Massachusetts, The Mediaeval Academy of America, 1953 [5 edición].

Asensio, Juan Carlos, "De la liturgia visigoda al canto gregoriano", en Gómez, M. (ed.): Historia de la música en España e Hispanoamérica. Madrid, Fondo de Cultura Económica, 2009, 21-76.

Breviarium Romanum ex decreto SS. Concilii Tridentini restitutum, S. Pii V, Pontificis Maximi, justi editum, Clementis VIII et Urbani VIII auctoritate recognitum. Paris, Apud St. Hilaire Blanc et S., 1846.

Casasnovas, Miquel Àngel y Sastre, Florenci, De Menorca a Istanbul: el saqueig turc de Ciutadella 1558. Ciutadella, Ajuntament de Ciutadella, 2010.

Colunga, A. y Turrado, L. (eds.), Biblia Sacra iuxta Vulgatam Clementinam. Madrid, Biblioteca de Autores Cristianos, 1946 [13 ${ }^{\mathrm{a}}$ edició, 2011].

Del Sol, Manuel, "Tradición hispana en las lamentaciones polifónicas del oficio de tinieblas: apuntes sobre tonus lamentationum hispanos en el siglo XVI", Revista de Musicología, 33/1-2 (2010), 247-267.

Escandell, Jaume, "Elasticitat i reformulacions melòdiques en les músiques de transmissió oral de la Mediterrània occidental: les sonades de flaüta i tambor d'Eivissa i Formentera", Anuario Musical, 65 (2010), 225-250.
Giuriati, Giovanni, "Parties fixes et 'à rallonges': le saltarello di Amatrice", en Lortat-Jacob, B. (ed.): L'improvisation dans les musiques de tradition orale. París, Selaf, 1987, 251-253.

Hardie, Jane Morlet, "Lamentations in Spanish sources before 1568: notes towards a geography", Revista de Musicología, 14 (1993), 220-250.

Julià, Gabriel, Els inicis del barroc a Menorca. L'església dels Socors de Ciutadella. Ciutadella, Nura, 1988.

Macchiarella, Ignazio, Il falsobordone: fra tradizione orale e tradizione scritta. Lucca, Libreria Musicale Italiana, 1995.

Nattiez; Jean-Jacques, Fondements d'une sémiologie de la musique. Paris, Union Génerale d'Éditions, 1975.

Pajares Alonso, Roberto L., Historia de la música en 6 bloques. Bloque 3. Difusión y notación. Madrid, Visión Libros, 2011.

Ruwet, Nicolas, "Méthodes d'analyse en musicologie", Revue Belge de Musicologie, 20 (1966), 65-90.

Sastre, Florenci, La Ciutadella de Menorca en el tránsito a la Modernidad. Ciutadella, Consell Insular de Menorca, 1982.

Trowell, Brian: "Fauxbourdon", en Sadie, S. (ed.): The New Grove Dictionary of Music and Musicians. London, Macmillan, 2001, vol VIII, 614-620.

Villalonga, Andreu y Fiol, Guillem, "El convent i les pintures murals dels Socors de Ciutadella. Aportacions documentals per a la seva conservació", Randa, 63 (2009), 5-18.

Zauner, Sergi, "Lamentaciones de Jeremías en Barcelona: tradición cantollanista pretridentina y apuntes sobre el contexto de interpretación del género polifónico a finales del siglo XVI", Nassarre, 26 (2010), 79-108.

Recibido: 12.12.2013

Aceptado: 02.11.2015 